\title{
General theory and foundations for calculating reinforced concrete structures of buildings and structures in modern construction
}

\author{
Rudolf Sanjarovskiy ${ }^{1, \mathrm{a}}$, Frieder Sieber ${ }^{2, \mathrm{~b}}$, and Tatyana Ter-Emmanuilyan ${ }^{3^{*}}$ \\ ${ }^{1}$ L.N. Gumilyov Eurasian National University, 11 Kazhymukan str., Nur-Sultan, 010000, Kazakhstan \\ ${ }^{2}$ Leibniz Institute for Interdisciplinary Studies (LIFIS), 16 Albert-Einstein-Strasse, Berlin, 12489, \\ Germany \\ ${ }^{3}$ Russian University of Transport (MIIT), 9, Obraztsova str., bldg. 9, Moscow,127994, Russia
}

\begin{abstract}
The theory which consists of two parts: short-term and long-term loading and of five inconsistent (or erroneous) theories that reject the fundamental properties of structural concrete and the principles of Eurocode and which is used in the calculations of various buildings and structures is analyzed.
\end{abstract}

\section{Introduction}

Methods. Using the mathematics rules, the principles of mechanics and the results of solid experiments, it was revealed that the analyzed theory contains a set of theories rejecting each other for various purposes, including erroneous ones; physically impossible skipping from one theory to another, change of various design schemes of a structure, unacceptable in the elastoplastic stage. There are mathematical errors in it: the fundamental concepts of classical and general theory of calculation are distorted; the design principle of the bearing capacity by the limit states, the process of continuous structures' loading established by Eurocode, is rejected; the properties of structural concrete fundamental according to Eurocode are replaced; the links to abstract experimental results are provided.

Results. The theory of mass application calculation analyzed in the article is accompanied by the necessary mathematical calculations and experimental estimates.

Reinforced concrete is the main building material of our time all over the world; residential, public and industrial buildings; underground space structures; transport, hydraulic and other structures made of it are erected.

According to the reinforced concrete properties, it is a very "complex anisotropic elastoplastic creeping material, composed of two materials different in their strength and deformation characteristics".

The analyzed theory consists of two parts: short-term and long-term loading [1]. And each part is based on arbitrary fundamental delusions caused by inconsistencies in the system (order) of general theory (strength of materials, theoretical mechanics, mathematics) [2].

The theory founders argue that the theory of calculating reinforced concrete "is conditioned not so much by the materials' properties but by the personal views and tastes of

\footnotetext{
* Corresponding author: tanya_ter@mail.ru
} 
the compilers" [17]. Misconceptions extend to the point that the analyzed theory calls itself a model of "the general theory calculation development in limiting states", declares itself "fundamentally equally suitable for different buildings and structures and for different materials."

The theory of long-term loading combines the rules violations of mathematics, mechanics, experimental data, and also states [13]: on an international scale, it is supposedly a new and most advanced format for calculating concrete creep for various buildings and structures, that it was "obtained after debate and cooperation between different schools and scientists at the world level with the international standardization organizations' assistance over the past 40 years ... ".

Reinforced concrete has a surprisingly unscientific, erroneous theory of calculation with huge volumes of use (annually in the world over 4 billion $\mathrm{m}^{3}$ ) and huge financial costs.

The work of a number of Round Tables held at the Peoples' Friendship University of Russia (RUDN) on the initiative and under the guidance of famous scientists: Bondarenko V.M., Krivoshapko S.N., Galishnikova V.V. was devoted to the problem of errors in the theory of reinforced concrete calculation (the last one took place in 2020), with a large number of participants, authoritative scientists from Russia and other countries. The main questions and judgments of the participants are caused by the following circumstance: in the scientific and educational literature, the theoretical essence of the foundations of reinforced concrete structures mixed in the calculations, which are not related to the properties of reinforced concrete and Eurocode, has not been properly studied and described. For example, in the main textbook the following is written: "Instead of the small sections hypothesis, the principle of plastic destruction is applied" ("to teach" means to transfer knowledge, scientific information).

\section{The analyzed theory features}

The analysis shows that the calculation theory of reinforced concrete structures, which are widely used (with long-term loading) all over the world, includes five that do not correspond to each other (among them are erroneous theories, the essence of which is set out below):

In the considered theory, they are mixed (instead of fulfilling the requirements of Eurocode):

(s.1) - theory of a structure that has no length and has a plastic hinge;

(s.2) - theory of an elastic-creeping column with initial deflection, which has no restrictions in stresses and deformations;

(s.3) - the theory of an infinitely elastic column from the "deformation theory", erroneously extended to the severe plastic deformations area;

(s.4) - distorted Euler problem;

(s.5) - deeply flawed theory of linear concrete creep.

(s.6) - The "new" theory is substantiated by an erroneous postulate about the method of "forming a plastic hinge".

The study shows that this scientific community and each theory individually does not correspond - neither to the properties of reinforced concrete, nor to Eurocode.

\section{The theory of a structure that has no length}

Here it is necessary to construct the corresponding theory of elastoplastic stability first (and there is no other way):

a) - to write the diagrams $\sigma$ - $\varepsilon$ for concrete and reinforcement to reject Eurocode and assume that these diagrams have unlimited yield pads 


$$
\left(\varepsilon_{t} \rightarrow \infty\right)
$$

b) - to use (and not reject) the hypothesis of flat sections, and find the values of the main vector and the main moment of the normal stress diagram.

c) - to write down the equilibrium equations of a compressed column, taking into account the presence of deflection.

d) - to consider the geometric aspect of the problem and associate the edge deformations of the section with the deflection.

e) - to formulate the condition and derive the critical state equation.

f) - to carry out the numerical studies and plot critical dependence curves.

The behavior of these curves depends on the column design model type. Let us consider two important cases: a column with initial deflection, longitudinal-transverse bending.

The design diagram of a column with initial deflection forms the basis of the theories ( $\mathrm{s}$. 2) and (s. 3), considered later (Fig. 3, a) within the framework of the linear theory. Here, for perception clarity, Fig. 1 shows the critical dependences curves of elastoplastic columns with initial deflection.

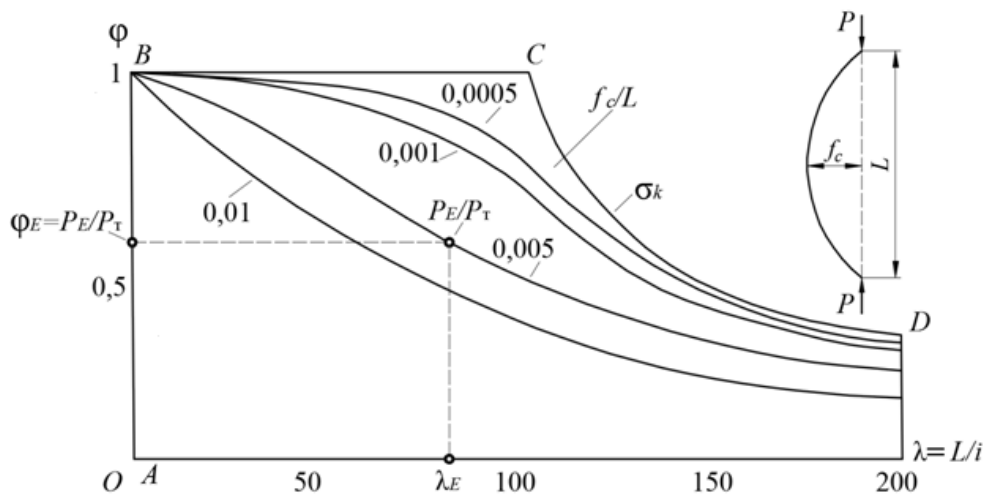

Fig. 1. Critical dependencies "force - flexibility - initial deflection" for an elastoplastic column.

In the case of the second design scheme - longitudinal-transverse bending, the curves of the critical dependences in the elastoplastic stage have a form similar to Fig. 1. Let us pay attention to the absence in two cases (different design schemes) of a plastic hinge according to the theory (s.1) [3-5], Fig. 2. In Fig. 1, point B can be attributed to the plastic hinge, which characterizes the fully compressed section ( $\mathrm{x}=\mathrm{h}$, in fig.2). In other words, the theory (s.1) is "fundamentally unsuitable" in the considered design schemes according to the analyzed theory developers' terminology.

About the plastic hinge. To get the theory (s.1), it is necessary to add two actions a) - f) to the above-described procedure:

g) - Select a special design scheme of the column, Fig. 2.

h) - Carry out mathematical passage to the limit

The plastic hinge is the ultimate point $(l \rightarrow 0)$ of the critical state curve $\left(\frac{d l}{d f}=0,1-\right.$ length, $\mathrm{f}$ - deflection) for stability for the columns made of concrete and steel with unlimited fluidity area, the edge deformations reach endless values in it, the zones of plastic tension and compression (satisfying the hypothesis of flat sections before the beginning of the passage to the limit) converge at the limiting point. And this local point, in a very private design scheme with unrealistic properties of compressed structures, is taken as the basis for the general theory of reinforced concrete calculation. 


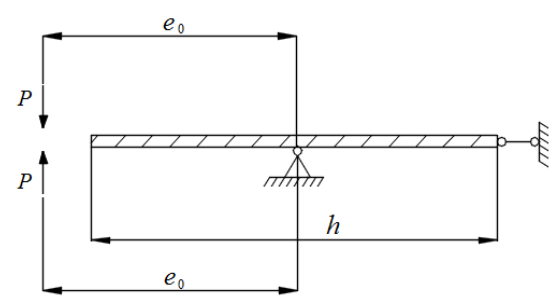

a)

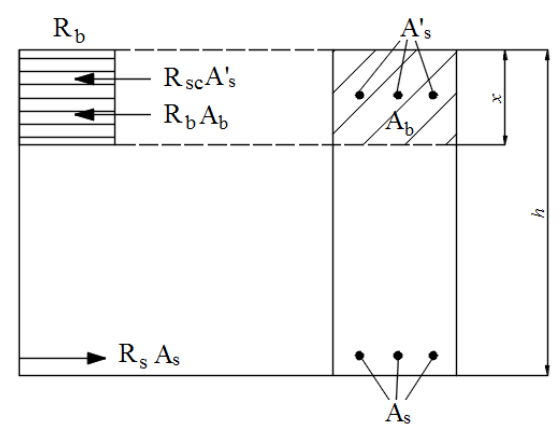

b)

Fig. 2. Column without length, for which a plastic hinge is possible: a) design scheme; b) cross-section, "ultimate forces" and stress diagram of concrete.

On the properties' unreality: the column has no length; the column section has the property of an absolutely rigid body. When passing to the limit, the elastic zone of the column section tends to zero, the plasticity covers the entire compressed zone height, and a degenerate model of the column is obtained in the form of one degenerate section, in which the force distribution system has one degree of freedom, with a generalized coordinate $x$, Fig. 2 .

So, the theory (s.1) has nothing to do with the calculation of real compressed structures, including reinforced concrete ones.

The foregoing also shows that under Eurocode conditions, when the diagrams $\sigma$ - $\varepsilon$ concrete and reinforcement are limited by ultimate deformation $\left(\varepsilon_{\mathrm{b} 2}, \varepsilon_{\mathrm{s} 2}\right)$, the theory $(\mathrm{s} .1)$ does not exist at all [6].

Finally, we consider the theory (s.1), which for the case of small eccentricities, described in the literature on reinforced concrete is very confusing. Here the compressed zone with the $x$ coordinate captures a part of the section of the lower reinforcement $A_{s}$, Fig. 2.b. In this fixture $A_{s}$ a local plastic hinge is formed, with a local main vector of forces and a main moment. The expressions for describing the local principal vector values and the principal moment of forces are very cumbersome due to the circular reinforcement cross-section. This cumbersomeness is overcome by two simplifications:

- the value of the local principal moment of forces in the reinforcement is considered negligible, see (8.10) in [5]

- the formula for the local principal vector is simplified by replacing a circular section with an equivalent section with a constant width, see, for example, (8.13) in [5].

External attractiveness and apparent simplicity made the main features of the theory invisible (to this day) (s.1): * (* a list of errors)

- the column has no length;

- the column section has the properties of an absolutely rigid body;

- endowment of concrete and reinforcement with an endless flow area;

- impossibility of obtaining (s.1) in another way, except for the mathematical passage to the limit.

\section{The theory of an elastic creeping column with initial deflection and the theory of infinitely elastic column from the "deformation theory"}

In the theories (s.2) and (s.3) reinforced concrete is endowed with new fantastic features that reject the theory (s.1): ** 
- no cracks in the sections;

- concrete works well in tension and compression;

- concrete and reinforcement are infinitely elastic materials

- concrete under tension and compression has infinite linear creep deformations (see also (s.5));

- stresses (in compression and tension) can exceed the ultimate strength of concrete and reinforcement for many times;

- theories are based on the hypothesis of "insignificant deflections" (in the terminology of S.P. Timoshenko), and in the calculation results the deflection infinitely "increases":

$f(\mathrm{p}) \rightarrow \infty$;

$f(\mathrm{t}) \rightarrow \infty, \dot{\mathrm{f}}(\mathrm{t})=$ const;

such contradiction is indicated as "the method is unsuitable" in general mechanics. For example, it leads to the creation of a (non-existent) critical force in flexural compression. In the analyzed theory, it is called "conditional critical force". In the educational literature, the features listed under the ${ }^{* *}$ sign are not indicated: there is only a formal indication "to multiply by the coefficient $\eta »$.

The design scheme for these theories is shown in Fig. 3.

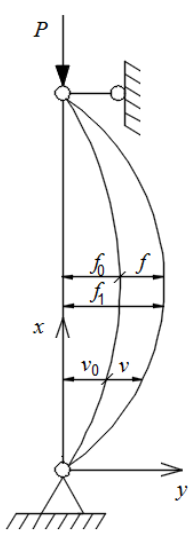

a)

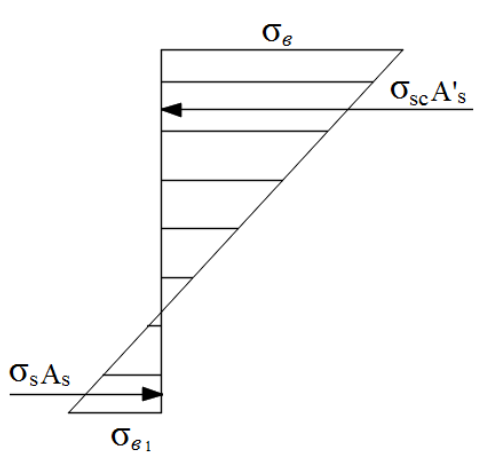

b)

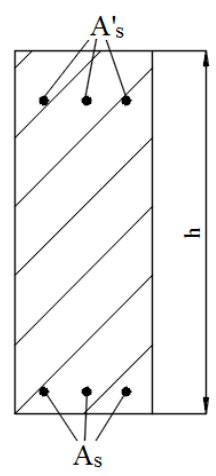

Fig. 3. Elastic or viscoelastic column with initial deflection: a) design scheme; b) cross section, unlimited stresses in concrete and reinforcement - no cracks

In the theory (s.2) "The relationship between stresses and strains is established by a formula based on a linear relationship between stresses and strains and on the superposition principle"

$$
\varepsilon^{*}(t)=\frac{\sigma^{*}(t)}{E(t)}-\int_{\tau_{1}}^{t} \sigma^{*}(\tau) \frac{\partial \delta(t, \tau)}{\partial \tau} d \tau
$$

where $\delta(t, \tau)=\frac{1}{E(\tau)}+C(t, \tau)$;

$C(t, \tau)$ denotes creep measure.

Here, and in what follows, the generally accepted designations of the constructed theory with errors are used. 
The theory (s.2) states: "It is known that in the case when the material of the rod has creep and aging (1), the stability problem of an elastic rod with an initial deflection $\left(y_{0}=f_{0}\right)$ and compressed by constant force $P$, is reduced to solving the equation»

$$
\frac{d^{2} y^{*}(x, t)}{d x^{2}}+\frac{P}{I}\left[\frac{y^{*}(x, t)}{E(t)}-\int_{\tau_{1}}^{t} y^{*}(x, \tau) \frac{\partial \delta(t, \tau)}{\partial \tau} d \tau\right]=\frac{d^{2} y_{0}}{d x^{2}}
$$

The deflection problem $f(t)$ is reduced to solving the "Volterra integral equation of the $2^{\text {nd }}$ kind"

$$
f(t)-\frac{E(t)}{1-\xi(t)} \int_{\tau_{1}}^{t} f(\tau) \frac{\partial \delta(t, \tau)}{\partial \tau} d \tau=f_{1}(t)
$$

where $\xi(t)=\frac{P_{\ni}(t)}{P} ; \quad P_{\ni}(t)=\frac{\pi^{2} I E(t)}{l^{2}} ; \quad f_{1}(t)=\frac{f_{0}}{1-\frac{P}{P_{\ni}(t)}}$

The critical state of a reinforced concrete column stability during concrete creep is determined by a criterion that is untenable according to Eurocode and surprising for the theory of reinforced concrete: the deflection of the column's middle section increases to infinity (with a constant rate of its growth). The structure of the formula for additional infinite deflection caused by concrete creep becomes identical to the infinitely elastic deflection structure according to the theory (s.3) (see, for example, the formula (8.13) in [5]). Only the value of the critical force is changed: instead of the short-term critical force of Euler, the concept of a long-term critical force is used, equal to the Euler force, divided by a coefficient depending on the concrete creep characteristics.

Special attention should be paid to three circumstances in the theory (s.2, s.3): **

- Euler's hyperbola is interrupted at the point $C$ (Fig.1), that is, on the site CB of the plastic domain, the concept of Euler's critical force (also a long-term critical force) is a fiction;

- With unlimited elastic properties, Euler's critical force does not exist for the compressedbent columns, Fig. 4, which additionally characterizes the theory inconsistency (s.2, s.3) from the point of view of Eurocode.

- Within the framework of any theory of creep, the theory (s.2) is incorrect for assessing the long-term resistance of reinforced concrete, since it endows concrete with fantastic properties of endless deflections.

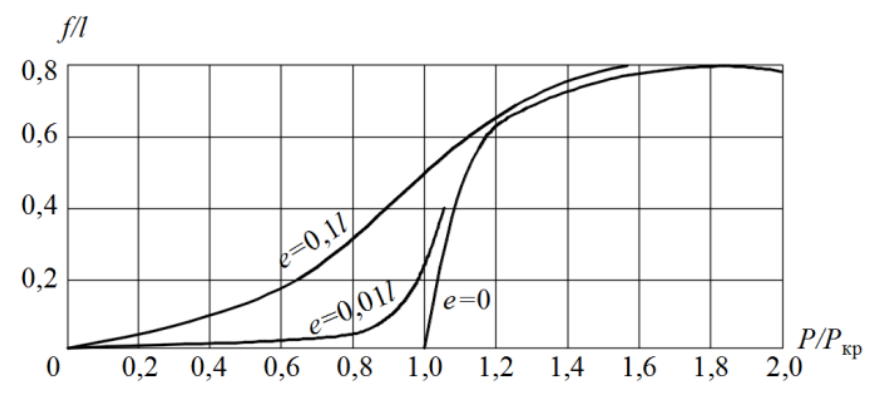

Fig. 4. The relationship between the deflection and the longitudinal force for the compressed-curved and centrally compressed $(\mathrm{e}=0)$ columns 
Each of the theories outlined is true only in its place in the general theory of the structures' calculation. So, the theory (s.1) is just one of the four lines - the boundaries of the region in the particular scheme of loading the elastoplastic stability - just one of many schemes in which there are no such boundaries at all (example, Fig. 1). Moreover, in this particular scheme, an ideally elastoplastic material with an infinite yield area is considered, that is, it is outside the rules of Eurocode 2 and is an error for the reinforced concrete theory [8].

Apparent novelty of the theory (s.1) and its erroneous attractiveness in the forties of the last century caused decisive actions to be introduced, and for the sake of these actions the essence of the theory of calculation of reinforced concrete was sacrificed, as evidenced by the hypothesis put forward by the theory (6) about the connection of theories (s.2, s.3) and theory (s.1):

"With eccentric compression ... the phenomenon of fracture proceeds almost the same as in bending, and the calculation is based on the same considerations and assumptions.

... In the interests of calculation simplicity, it is even more desirable than in the case of bending symmetric sections, to assume ... that the section behaves elastically up to the formation plastic hinge".

So, let us consider the sequence of skipping from one theory to another. We suppose initially there is a reinforced concrete column with the given properties of the bearing capacity. Let us apply the theory to it (s.1), we get a strongly overestimated bearing capacity after the calculation execution.

For this reason, we turn to the theory (s.3) or (s.2), replacing the design scheme of the theory (s.1) for the design diagram of the column with initial deflection. It would be possible to use the theoretical data for the sample in Fig. 1, but it does not.

The process of loading an infinitely elastic column is considered, at the end of which the cited hypothesis is extracted and the transition from (s.3) for (s.1) in the form of the following amazing actions:

- The length of the infinitely elastic column disappears abruptly; only one section with a linear stress diagram without a crack remains.

- Elastic stress diagram, according to Fig. 3, instantly turns into a diagram of the plastic hinge stresses, according to Fig. 2.

- Initial deflection $\mathrm{f}_{0}$ elastic column from (s.3) instantly becomes a given eccentricity in theory (s.1).

- Boom of elastic column $\mathrm{f}$ theory additional deflection (s.3) turns into eccentric theory (s.1), which is called additional eccentricity and the appearance of which destroys the theoretical essence of the plastic hinge, described above, as the essence of a column that has no length.

- A "new" scientific essence of the general theory appears in the form of a plastic hinge that has no length, but has a deflection; sum $e_{0}+f$ becomes the calculated eccentricity $e_{0} \eta_{1}$ in theory (s.1).

Based on the "new" entity, the bearing capacity of the given reinforced concrete column is again calculated: the calculation results overestimate the bearing capacity of the given column again.

Even more paradoxical is the connection into one theory of a plastic hinge along (s.1) with time-varying deflection theory (s.2). The "new" scientific essence in this case is an amazing continuous change in the longitudinal force of the column, which occurs over time, as well as the continuous phenomenon of skipping.

The theory of reinforced concrete in the "new" scientific essence acquires dual properties in many circumstances and parameters, which makes it possible to change the meaning of these parameters, to conduct unscientific discussions. 
For example, in the theory of a plastic hinge (s.1) section stiffness $D=E I$ is not needed. But for the "correction" of the reinforced concrete analyzed theory, the "new" scientific essence makes it possible to use and distort this concept.

\section{The distorted Euler problem}

In the classical Euler problem on the column stability, the theory (s.4), representing the differential equation of bending has the form:

$$
D \frac{d^{2} v}{d x^{2}}=-P v
$$

As already noted, on the site $\mathrm{BC}$ according to Fig. 1, this equation does not exist in the plastic region. Like rigidity in the theory (s.1); there is no Euler force either. The "new" scientific entity not only introduces Euler's force, which does not exist here, but also distorts its meaning by inventing Euler's force that depends on eccentricity $\mathrm{e}_{0}$

$$
N_{c r}=\frac{\pi^{2} D\left(e_{0}\right)}{l^{2}}
$$

*** - The general theory is "corrected" by this technique: the critical forces of a reinforced concrete column $\left(\mathrm{N}_{\mathrm{cr}}\right)$ for short-term loading, and $\mathrm{P}_{\mathrm{p}}$ under prolonged loading, impossible with eccentric compression (see Fig. 4.), are declared as not only possible, but also "undergo evolutionary development" in the form of an absurd dependence on eccentricity.

Experimental estimates of the calculating compressed reinforced concrete structures' results according to the analyzed theory, given by well-known scientists in the publications in recent years, are $\pm 50 \%$, testifying that unscientific nature and non-compliance with Eurocode, in addition to political aspects, give low reinforced concrete economic efficiency.

\section{Erroneous theory of concrete linear creep}

In the theory (s.5), which is a world theory, the Volterra integral equations representing the creep of concrete with its non-stationary and non-linear properties have fictitious nuclei that violate the envisaged mathematical order of their construction: as a result, concrete forms an erroneous set of fictitious forces that incorrectly form creep deformations [7-10].

We have found that all the main provisions of the theory (s.5) grossly violate the rules of higher mathematics, the principles of mechanics, Eurocode requirements and results of solid experiments. Among them:

- the theory foundation, its principle of superposition violates the differentiation rules of functions. This violation is accompanied by a crafty justification [13] that "the superposition principle is inherent in Volterra's theory": as a result, erroneous kernels of integral equations are constructed. There are a number of other ridiculous "mathematical" justifications for this principle;

- "There is no linear creep," according to famous scientists S.V. Aleksandrovsky and P.I. Vasilyev [11]., giving the experimental data on Fig. 5;

- instantaneous concrete deformations, nonlinear according to Eurocode, are declared as elastic, which is justified by the non-existent experiments; 
- the non-stationarity of instantaneous deformations is incorrectly identified with the Maxwell model, and is described using the superposition principle, introducing an error up to $300 \%$;

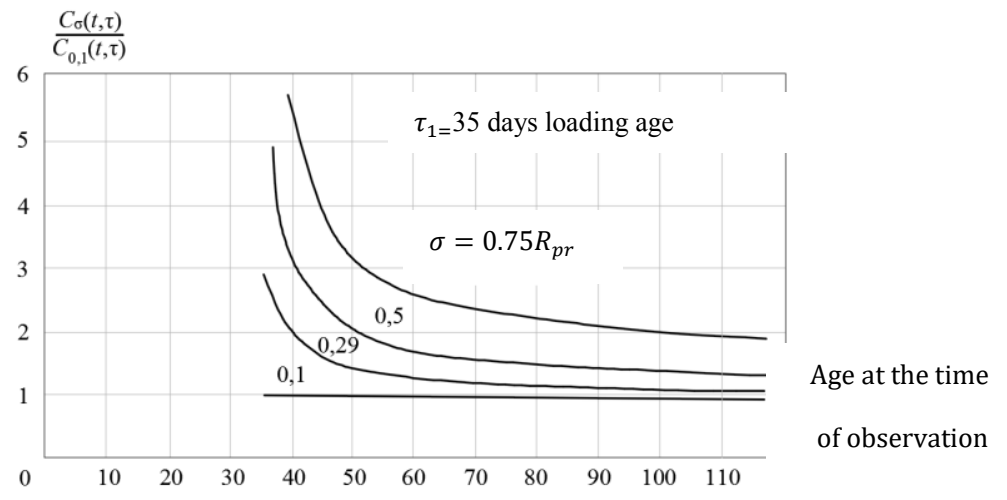

Fig. 5. Changes in the specific creep strains ratio at different initial stress levels $C_{\sigma}(t, \tau)$ to specific creep deformations at the initial stress level $\mathrm{C}_{0,1}(\mathrm{t}, \tau)$

- alteration of instantaneous nonlinear concrete properties, creep properties (minute creep, chain models, fast-flowing creep) inadmissible in mechanics is carried out; this leads to the resistance forces appearance proportional to acceleration, creates a violation of the forces action independence principle (the fourth axiom), distorting the entire theory;

- "Algebraization" of the creep theory rejects the basic equation of Newtonian mechanics, returns to the level of Aristotle mechanics; this was repeatedly emphasized by N.H. Harutyunyan and S.V. Aleksandrovsky.

In the analyzed theory, it is also possible to find more absurd situations when the conventional (for reinforced concrete) theory (s.2) elastic-viscous stability of a compressed rod with initial deflection, with infinite stresses, with its value of long-term critical force $P_{p}=\frac{\pi^{2} E I}{l^{2}(c+1)}$, where $c=\varphi_{\infty}$ becomes the theory for calculating the limiting state of reinforced concrete shells with the cracks under prolonged loading. It is included in the methodological recommendations, in the figure of the elastic modulus $E_{p}=\frac{E}{c+1}$ ( $c=\varphi_{\mathrm{c}}=2$ ), the limiting characteristic of ordinary concrete creep of, is masked by empirical expressions, which is especially clearly seen from the problem of calculating the structures of the Transvaal Park.

\section{Conclusion}

The analyzed theory is preceded by an explanation in the form of two Provisions in a number of works as well as in the norms: I - it is necessary to use a nonlinear deformation model (sign); II - it is allowed to calculate on the analyzed theory basis (with different names: calculation by limiting forces; calculation by the destruction stage or by the plastic destruction principle; limiting equilibrium method; calculated limit states method).

One of the norms developers in 2011 warned that an ordinary designer would not be able to use Regulation I: "The deformation model of force resistance is mainly implemented through the computing systems, so here a number of formal procedures arise, for example, stability, the solution accuracy estimation. The lack of tools is also due to the multi-iteration 
process of the solution, especially as the acting force approaches the bearing capacity ... The results depend on the choice correctness of the initial (calculated) state diagrams". Russia joined WTO and is obliged to comply with the Eurocode requirements.

Since Eurocode prohibits changing its Principles and Rules of Application, and an ordinary designer will not be able to apply Regulation I, we come to the misconception that Regulation II corresponds to Eurocode. In the educational literature in this regard, it is stated: "Instead of the hypothesis of flat sections, the principle of plastic destruction is applied"; "The proposal to determine the bearing capacity by the limiting ("plastic") state for decades outstripped the world practice in this matter"; "In the calculation models of Eurocode, there is also a calculation for the ultimate effort" - which misleads the specialists. Comparing the national standard and European standards, A.A. Gvozdev et al. [12] pointed out their significant difference in the principles and methods of calculation and, in particular, concerning "the calculation of normal ... sections, taking into account the columns flexibility influence and the load duration".

The unscientific nature of the reinforced concrete analyzed theory in certain aspects and at different times was pointed out by the authoritative scientists: Skramtaev B.G., Keldysh V.M., Nikitin G.V., Rzhanitsyn A.R., Geniev G.A., Drozdov P.F., Tal K.E. and others. The average response to criticism sounded evasive: "The calculation scheme choice is determined by considerations of a didactic nature". After the approval of Eurocode, the unscientific nature and inconsistency with Eurocode of the analyzed theory became obvious.

The problem of unscientific concrete creep theory is indicated by the design practice negative results, including the world experience in the design of unique structures with RAMBOLL structures (Great Britain) [13]; fib president Gordon Clarke warns: "accurately predicting the impact of creep ... has a highly controversial character "; we have established the reasons for the unscientific nature of this theory - among them are mathematical errors and violation of the classical mechanics principles $[7-9 ; 14]$, we have also developed a new nonlinear theory of concrete creep, not yet published, supplementing the general theory [15].

The results of the reinforced concrete calculation theory analysis, as well as the essence of mathematical errors in the theory of concrete creep were reported and discussed at theinternational symposium in Belgium in 2018 [16], and at the international conference in Moscow in 2014 [13].

\section{References}

1. A.D. Beglov, R.S. Sanjarovskiy, V.M. Bondarenko, European standards and nonlinear theory of reinforced concrete, Concrete and reinforced concrete are ways of development, Scientific works of the II All-Russian (international) conference 1, 119131 (2005).

2. ACI 209.3R-XX, Analysis of Creep and Shrinkage Effects on Concrete Structures, Final Draft, M.A. Chiorino (Chairm. of Edit. Team), ACI Committee 209, March 2011.

3. V.N. Baykov, E.E. Sigalov, Reinforced concrete structures (Moscow, 1991).

4. V.M. Bondarenko, D.G. Suvorkin, Reinforced concrete and stone structures (Moscow, 1987).

5. Set of rules BC 63.13330.2012. Concrete and reinforced concrete structures. Basic provisions. Updated edition SNiP 52-01-2003, Moscow, 2012.

6. EN 1992-2 2004, Eurocode 2: Design of constructions.

7. R.S. Sanjarovskiy, M.M. Manchenko, Structural mechanics of engineering buildings and structures 3, 25-32 (2016). 
8. R.S. Sanjarovskiy, T.A. Ter-Emmanuilyan, M.M. Manchenko, Structural mechanics of engineering buildings and structures 14 (2), 92-104 (2018). DOI: 10.22363/1815-52352018-14-2-92-104.

9. R.S. Sanjarovskiy, M.M. Manchenko, M.A. Gadjhiyev, T.T. Musabayev, T.N. TerEmmanuilyan, K.A. Varenik, Structural mechanics of engineering buildings and structures 15 (1), 3-24 (2019). DOI: 10.22363/1815-5235-2019-15-1-3-24.

10. R.S. Sanjarovskiy, M.M. Manchenko, Structural mechanics of engineering buildings and structures 6, 25-36 (2017).

11. Creep and shrinkage of concrete and reinforced concrete structures. State of the problem and development prospects, GOSSTROY USSR, Concrete and Reinforced Concrete Research Institute (Moscow, Stroyizdat, 1976).

12. A.A. Gvozdev, Yu.P. Gushya, E.A. Chistyakov, Concrete and reinforced concrete 5, 2425 (1979).

13. Concrete and reinforced concrete - a look into the future. Scientific works of the III AllRussian (II International) conference on concrete and reinforced concrete (Moscow, 2014).

14. R.S. Sanjarovskiy, T.A. Ter-Emmanuilyan, and M.M. Manchenko, CONCREEP 10, 238-247 (2015)

15. R.S. Sanjarovskiy, Creep stability of building structure elements (Publishing house of Leningrad State University, 1984).

16. Life-Cicle Analysis and Assessment in Civil Engineering, Proceedings of the Sixth International Symposium of Life-Cicle Civil Engineering (IALCCE), 2018, 28 October, Ghent, Belgium.

17. The theory of concrete and reinforced concrete structures calculation, Moscow, 1949. 